\title{
Energy Aware Power Save Mode based AODV Routing for IEEE 802.11s Wireless Mesh Networks
}

\author{
S.P. Shiva Prakash \\ Research Scholar \\ JSS Research Foundation \\ SJCE, Mysuru
}

\author{
T.N. Nagabhushan \\ Member, IEEE \\ Department of Information Science and Engineering \\ SJCE, Mysuru
}

\author{
Kirill Krinkin \\ Adjunct Professor \\ Saint-Petersburg Electrotechnical University "LETI" \\ Saint-Petersburg, Russia
}

\begin{abstract}
Owing to the self organizing, self configuring and self healing capabilities, Wireless Mesh Nwetworks (WMN) have emerged as most powerful architectures in recent times. Wireless Mesh Networks operate with limited battery resources. In 802.11s, a mobile station(STA) can act as router as well as client that results in increased energy consumption during routing process. The degradation of energy at STA can result in low network performance due to relay STA failure. In order to increase network performance there is a need to reduce the energy consumption rate of STA. To save energy, $802.11 \mathrm{~s}$ has introduced a mechanism called power save mode(PSM) which switches STA mode from active to light sleep or deep sleep mode when STA is not involved in transmission. This ensures saving of energy at each STA during routing process. Several models have been proposed by researchers to improve the energy saving mechanisms in $802.11 \mathrm{~s}$. Adhoc On Demand Distance Vector (AODV) routing algorithm is used for routing purpose in WMN. In this routing protocol, regular usage of the same node during routing may lead to battery failure and result in low network lifetime. Hence in this work we propose a novel Energy Aware Power Save Mode-AODV (EAPSM-AODV) routing model that switches among power save modes based on battery status of a node before participating in AODV routing. Our proposed routing model refers to the mechanism that takes available remaining energy and energy draining rate of a node into account to decide on relaying traffic. It consist of four units namely Routing, Path choosing, Mode selector and Energy units. Experiments have been conducted using NS3 simulator by considering various situations such as keeping nodes in static position and moving nodes dynamically in a simulation area. The results shows that our proposed model significantly improves network performance and increase in network lifetime.
\end{abstract}

\section{General Terms}

Power Save Mode, AODV Routing

\section{Keywords}

PSM, AODV, IEEE 802.11s, WMN, Energy Aware

\section{INTRODUCTION}

Wireless Mesh Networks (WMNs) consist of mesh routers and mesh clients where mesh routers act as a backbone of WMNs with minimum mobility. They provide network access for both mesh and conventional clients. The WMN can integrate with other network such as the Internet, cellular, IEEE 802.11, IEEE 802.15, IEEE 802.16 , sensor networks, etc., which can be done through the gateway and bridging functions in the mesh routers. Mesh clients can be either stationary or mobile and can form a client mesh network among themselves and with mesh routers [5].

In wireless mesh networks, battery plays a major role in deciding the efficiency of routing protocols. Since battery is a limited resource of energy, we must be very careful in the design of energy efficient routing protocols. Any node in a WMN can be in the state of transmission, reception or relaying of packet during transmission. It is known that the battery energy draining rate is higher during transmission state compared to idle state. Hence IEEE 802.11s standard introduced a mechanism called Power Save Mode (PSM) in which mesh station (STA) acts in three different modes such as active, light sleep and deep sleep mode. A STA must change its mode to active from light sleep mode before involving in transmission. Otherwise it has to be in the sleep mode. This mechanism reduces energy consumption of STA and increases network life time. In PSM based routing, mesh nodes change their state from light sleep mode to active mode whenever there is a request from beacon mesh node to transmit or receive or relay a packet. Figure 1 shows PSM based routing in static nature of nodes. Switching of modes for six different mesh stations STA-A, B, C, D, E and F. STA-A, STA-B, STA-E and STA-F are in Active mode, STA-C is in Light Sleep mode and STA-D is in Deep Sleep Mode. Mesh STAs creates a link and maintains a link specific power mode towards each peer STAs. It also tracks the power mode of each peer and only exchanges data frames with its peer. A link consists of two mesh STAs and both STAs have their own independent power mode for 


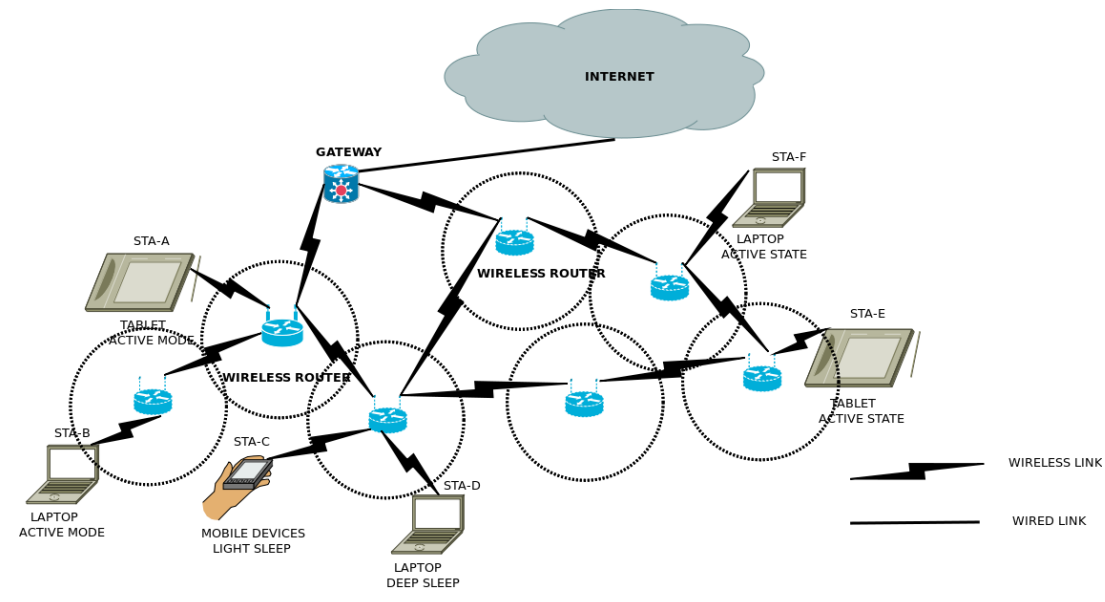

Fig. 1. PSM based routing under static conditions

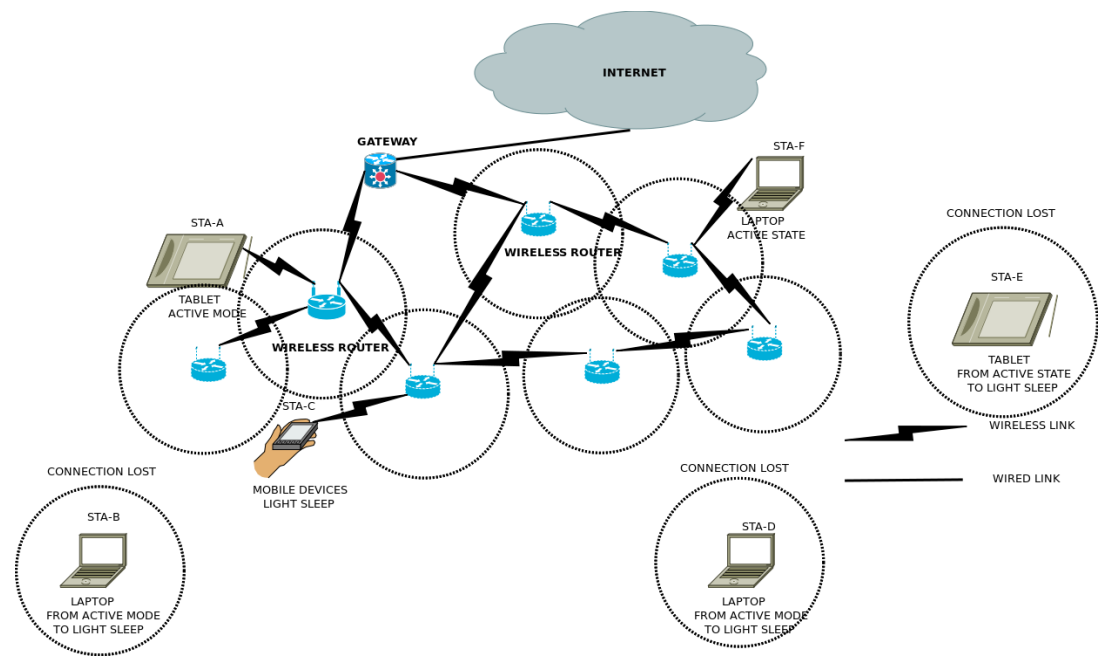

Fig. 2. PSM based routing under mobility conditions

each other. A STA can operate in any of the three power modes for a link. A single mesh STA can serve its various peers in different power modes at the same time. Figure 2 shows the PSM based routing under mobility conditions of node. STA-A will remain in Active mode as it will be within the communication range whereas STA-B, STA-D and STA-E will change their modes from Active to Light Sleep mode due to loss of connection during mobility. In light sleep mode for a link, a PSM STA wakes up periodically to listen to all the beacons of the peer STA. On the other hand, in deep sleep mode for a link, a PSM STA may not wake up to listen to all the beacons. In active mode operation, a STA remains in awake state all the time. If a STA works in either light sleep or deep sleep mode for any of its peer links, STA will alternate between awake and doze states for that link, as determined by the frame transmission and reception rules [6]. A Peer Service Period (PSP), which is an agreed contiguous time period is used to exchange buffered frame in a link if the receiver STA operates in PSM.

Most of the related works presents energy aware routing protocols in WMN. In this work we extend the work proposed by Shiva et. al., [12] in which the PSM is triggered based on the battery resource of a node. We adapt EAPSM to AODV routing protocol during decision making process of a node. A critical issue in wireless mesh network is to maintain network activities long time by keeping energy efficiently over all nodes in the network. Since routing process in wireless mesh network consumes energy at each node, there is a need to find the energy required by the node before involving in routing process. Further depending on the energy status, switching between PSM will result in choosing best path during routing. The rest of the paper is as follows: Section 2 discusses the related works carried out by various researches, Section 3 introduces the proposed model, Section 4 presents the algorithm, Section 5 shows the results and discussion and Section 6 presents the conclusion.

\section{RELATED WORKS}

In this section we present the related works on enhancing the network lifetime of WMN.

A WMN typically consists of networks of access points, which are connected through a wireless backbone. Research focus has been diverted in finding ways to minimise the power consumption of these networks. Lamia Romdhani and Christian Bonnet 
[8] introduced method for optimizing AODV by considering the energy consumption speed. Praveen Gupta, Preeti Saxena [10] showed that, the huge gap between the nodes consume more battery power. Hence, the amount of power consumed while transferring the data depends on distance between two nodes. Adeel Akram, Mariam Shafqat [1] introduced a modified AODV routing protocol for battery and frequency optimization to be employed in Wireless Mesh Networks (WMN). Since routing is an essential function of such multi- hop networks, T. A. Le and M. R. Nakhai [7] showed a method to reduce power consumption by avoiding long range communication in favor of multi-hop transmissions. Jharna Chokhawala, Albert Mo Kim Cheng [6] discussed that there are different routing algorithms which consider metrics like hop count and expected transmission count but there is a chance of some repeatedly used node that may get drained earlier. Hence, they proposed an algorithm with one more metric called energy for path selection. Mhalanga et al. [9] introduced an energy optimization based path selection algorithm for IEEE 802.11s WMNs that will maximize the network lifetime addressing the self configuration and cost effectiveness constraints of WMN.

Authors Xi Chen et.al.,[13] proposed an enhancement to the default 802.11 Power Save Mode (PSM), called M-PSM, it provides the additional power-saving opportunities by considering user mobility and detailed traffic condition. Further authors have compared the performance of the proposed M-PSM scheme against the default 802.11 PSM scheme. A.M. Akhtar et.al., [2] came up with Energy-efficient adaptive routing in wireless ad hoc and mesh networks and Proposed a power saving routing (PSR) algorithm that takes into account the multipath fading effect. Authors have considered the parameters such as the no of nodes and the APAR-M1 and APAR-M2. APAR (Adaptive power-aware routing) where APRMM1 was the first metric used to calculate the next hop destination, that is when it simply compares the channel gains and APRM-M2 compares the channel gain to remaining distance ratios to calculate the next hop destination. Authors Yifei Wei et.al., [13] presented a technical paper on Energy Saving Dynamic Relaying Scheme in Wireless Cooperative Networks Using Markov Decision Process by Proposing a dynamic relaying scheme based on relay selection and physical-layer power control with the objective of minimizing the energy consumption for data transmission. Authors have considered the simulation environment with two hop-relay network with source, destination and $\mathrm{N}$ relay nodes. Each node operates in half duplex mode. Communication is divided into Transmission phase, Channel estimation and relay selection phase and Retransmission phase. Authors Alves, R.L et.al., [4] presented Persistence of Routing Tables in Wireless Mesh Networks with Duty Cycling by proposing the mechanism referred as PORT (Persistence of Routing Tables) which keeps track of routing table entries while nodes are in the energy saving mode. Authors Akhtar, A.M et.al., [3] Proposed a cooperative routing algorithm. It considers the active radio electronic power consumption while selecting the minimum power route from a given source to destination. Author estimates the link cost model, system power consumption model and power gain.

Most of the work carried out consider only hop count as a parameter to AODV routing in WMN. It is on-demand and discovers route only when they are needed. It incorporates distance vector routing protocol. In WMNs, battery energy at the nodes is a very limited resource that needs to be utilized efficiently. The failure of some node's operation can greatly reduce performance of the network and even affect the basic availability of the network. The potential problem in current protocols for WMNs is that, they find the shortest path and use same path for every communication. However, this is not the best thing to do for network lifetime. Using the shortest path frequently can lead to energy depletion of the node along that path and in the worst case may lead to network partitioning or network failure. To overcome this, authors T.N. Nagabhushan et.al [11], have proposed a novel approach that selects path based on the remaining energy and energy draining rate metrics of node during AODV routing. Further authors S.P.Shiva Prakash, et.al [12] proposed a model which triggers PSM based on the energy constraint. Hence, In this work we extend the works of [11], [12] and present a novel AODV routing protocol which uses energy aware PSM during routing.

\section{PROPOSED MODEL}

In this section we present our proposed model.

Figure 3 shows the proposed EAPSM-AODV routing model that uses PSM status of node. It has four main units namely Routing, Path choosing, Mode selector and Energy. The working of model is as follows:

Routing: It has four sub units called transmitter, receiver RREP, RREQ which interacts with a processor. The processor receives a stream of data from the path choosing unit. When a RREQ is received, the processor sends the data to the energy unit for processing and selection of the mode. If the routing unit receives a RREP it processes and analyses all active nodes with respect to the three metrics and chooses appropriate path and transmits data. The node with PSM listens for RREQ messages in the mesh network. When it receives RREQ it may or may not reply to requested node depending on the drain rate and remaining energy of battery. Unlike conventional PSM, proposed model uses an adaptive energy threshold method where the energy required for a packet is calculated before participating in transmission. It determines the amount of energy required by a node to process the packet. It sets the threshold energy value required for a node to participate in routing process. This performs as a deciding factor for a node to be involved in a transmission.

Energy: It calculates the energy consumed by a node during static and dynamic in nature. It contains two sub units energy consumption calculator and remaining energy calculator. Static energy consumption calculator calculates the energy consumed by a node considering only packet size and data rate as parameters using equations [12]. Remaining energy calculator calculates the remaining energy of a node by calculating the differences between initial energy and the current energy.

Mode selector: It takes the calculated remaining energy and remaining energy threshold as input to schedule PSM. It compares the remaining energy of a node with the remaining energy threshold required by the node to participate in transmission. If the remaining energy of a node is greater than the minimum energy threshold required by node to participate in transmission, the scheduler changes nodes current PSM state from light sleep mode to Active mode otherwise PSM of a node remains in Light Sleep mode. If the remaining energy of a node gets drained completely and reaches NULL or value 0 , then scheduler changes the current PSM state of node to Deep Sleep mode. If the resulting mode is active it sends a RREP to the source node. Suppose the packet energy is different, the estimated DR and RE threshold values will be set accordingly to the packet energy. Thus this adaptive nature of the threshold value makes it more efficient. 


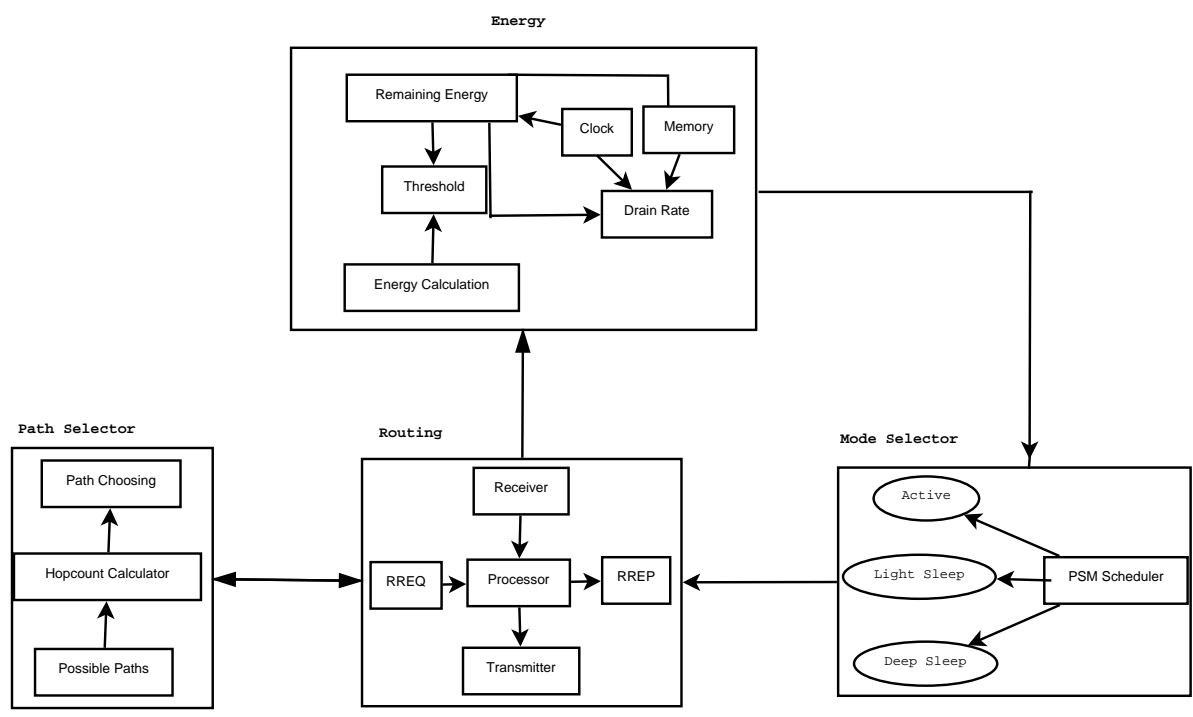

Fig. 3. Proposed EAPSM-AODV model

Path choosing: It chooses the best path using basic three parameters like hop count, transmission count and expected transmission time along with two additional parameters like draining rate (DR) and remaining energy (RE) and the status of the receiver node. The path choosing unit identifies all possible paths from source node to destination and a hop counter to calculate number of hops in all these paths with minimum number of hops is choosen. Similar operation is performed for all possible paths to the destination and a suitable path is choosen and sent to the routing unit.

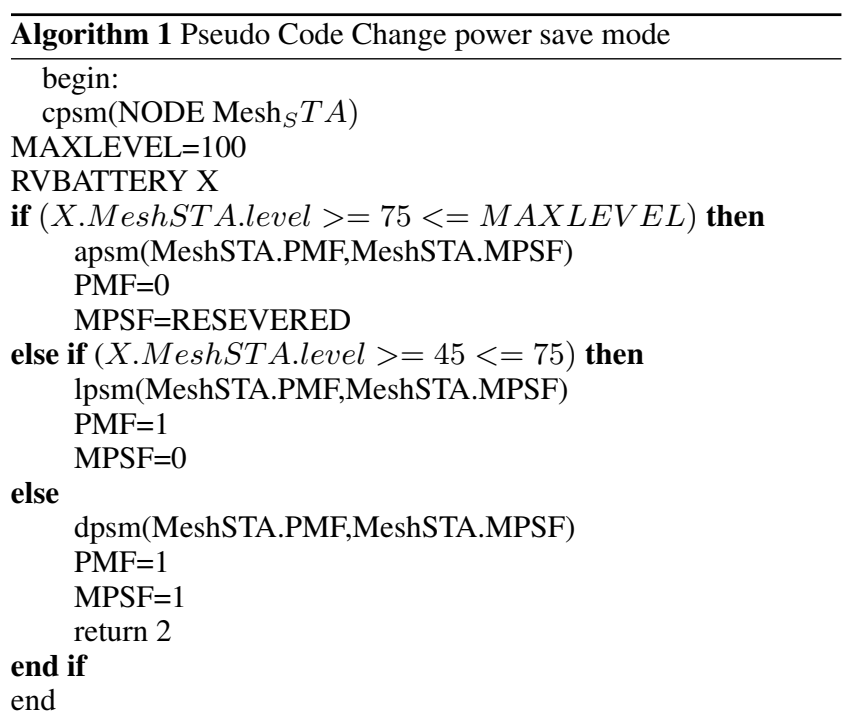

Algorithm 2 presents the pseudo-code used to trigger between PSM during AODV routing process. Any node that receives RRREQ message to participate in routing, it checks its PSM before participating. Only a node with Active PSM status can participate in routing by sending RREP message. The triggering of PSM is made based on the battery status of a node. All the nodes having Active PSM status will be represented in routing table of AODV. Energy aware AODV routing protocol is used to choose the best path during routing process. A node having minimum battery draining rate and maximum remaining energy will be choosen while selecting best path. 


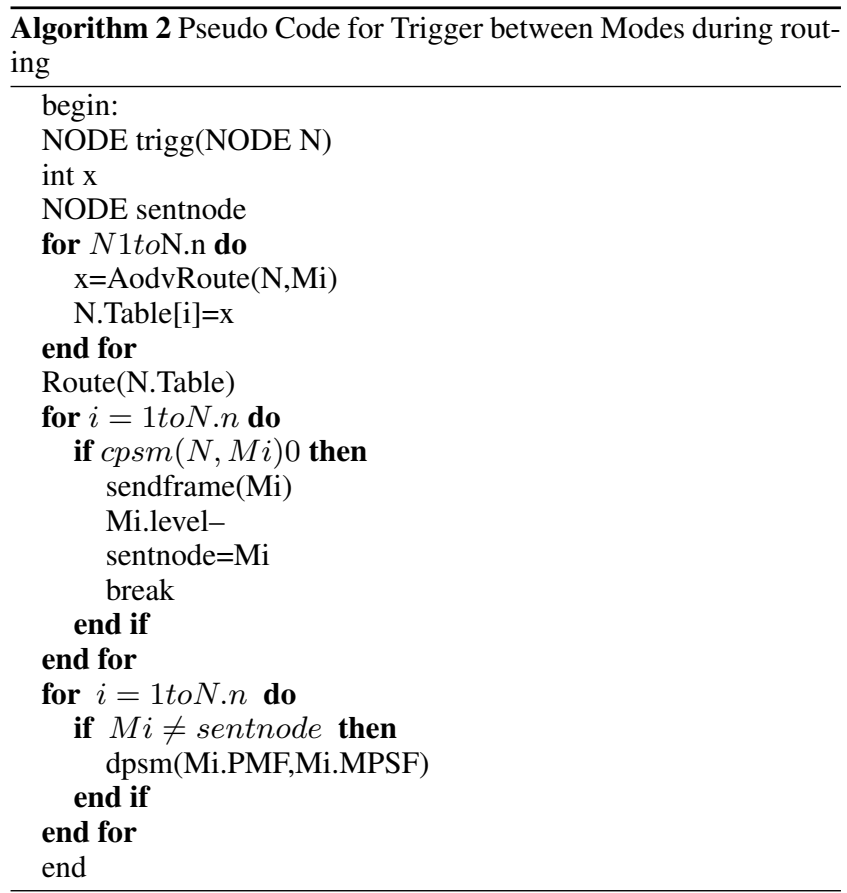

\section{RESULTS AND DISCUSSION}

In this section, we present the results obtained during experimentation conducted using Network Simulator NS3.

\subsection{Simulation Setup}

The simulation was setup for the proposed model by creating 5 X5 mesh dynamically moving around the specific area in wireless mesh network using NS3. RandamWayPoint mobility model is used to move the nodes in a simulated area. IP address was assigned to every node's device interface and was set with the AODV routing algorithm. The RV battery model available in NS3 simulation tool is installed to all the nodes. The available energy and draining rate is calculated using the equations defined in paper [12]. The source and destination nodes have been selected among the 25 available nodes randomly. Each node is connected via UDP. The following parameters are set to AODV routing algorithm as shown in Table 5.1

\subsection{Results}

The effciency of proposed protocol is tested by using the parameters defined in Table 5.1. The performance metrics we considered are packet delivery ration (PDR), packet reception time, packete transmission time, delay and PSM mode of nodes.

Figure 4 depicts the comparison of average delay obtained during simulation. It can be noticed that our proposed model results in less delay compared to existing model. This is due to the usage of adaptive routing metric which choosed best path during routing. Figure 5 shows the comparison of Transmission time of existing and proposed models. It can be noticed that the proposed model takes less transmission time compared to existing model.

Figure 7 shows the PDR comparison between the proposed and existing models in static nature of nodes. It can be noticed that proposed model results in $100 \%$ PDR compared to existing model.
Table 1. Simulation Parameters

\begin{tabular}{|c|c|}
\hline Parameters & Values \\
\hline Area & 300 X 1500 \\
\hline Nodes & 25 \\
\hline Nodes speed & $40 \mathrm{~m} / \mathrm{s}$ \\
\hline Simulation Time & $400 \mathrm{~s}$ \\
\hline Traffic Sources & 12 \\
\hline Traffic Type & CBR \\
\hline Packet Size & 312 bytes \\
\hline Start of Traffic & $30 \mathrm{~s}$ \\
\hline End of Traffic & $1.4 \mathrm{~W}$ \\
\hline Transmission Power & $1.0 \mathrm{~W}$ \\
\hline Reception Power & $0.01 \mathrm{~W}$ \\
\hline Idle Power & $10 \mathrm{~J}$ \\
\hline Initial Energy & Light Sleep \\
\hline Initial PSM Mode & 0 \\
\hline Initial Transmission Mode & \\
\hline &
\end{tabular}

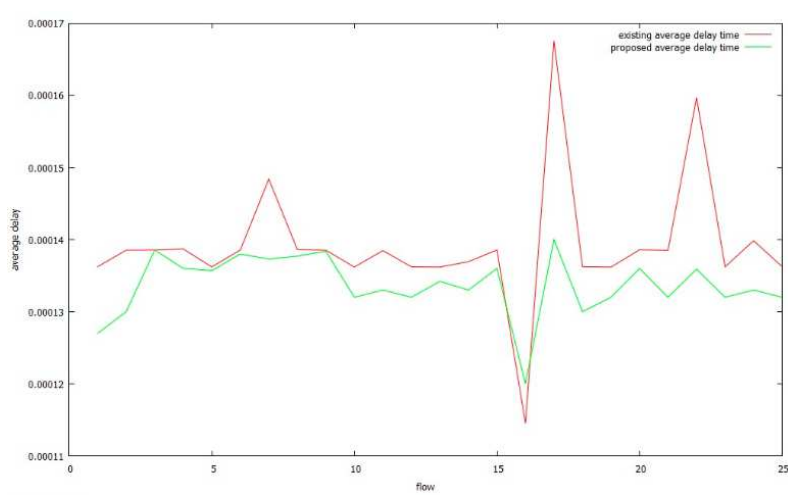

Fig. 4. Comparison of average delay between existing and proposed model

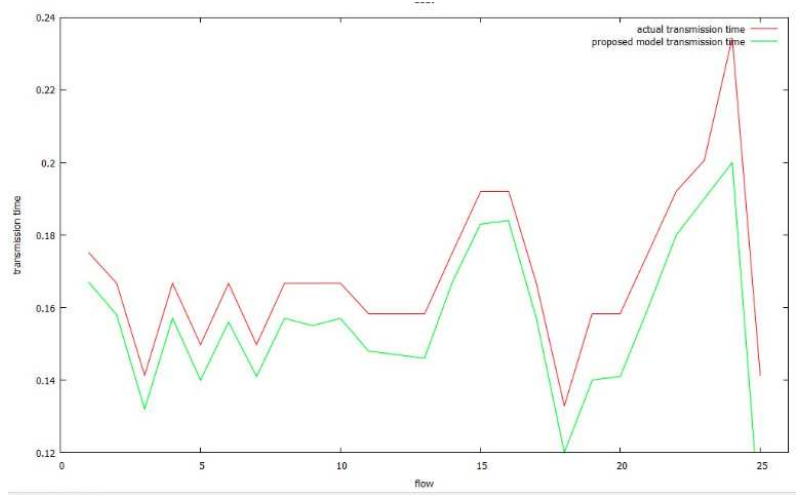

Fig. 5. Transmission Time

This is due to the consideration of metrics such as remaining energy, draining rate and PSM mode of a node during routing process. Figure 8 shows the variations in the mode of a node at different instant of time. The Tx, Rx, Rel and idle represents transmission, reception, relay and idle mode of node respectively. It can be noticed that in the proposed model nodes will remain in idle mode for a long duration which results in energy saving of a node there by increasing network lifetime. 


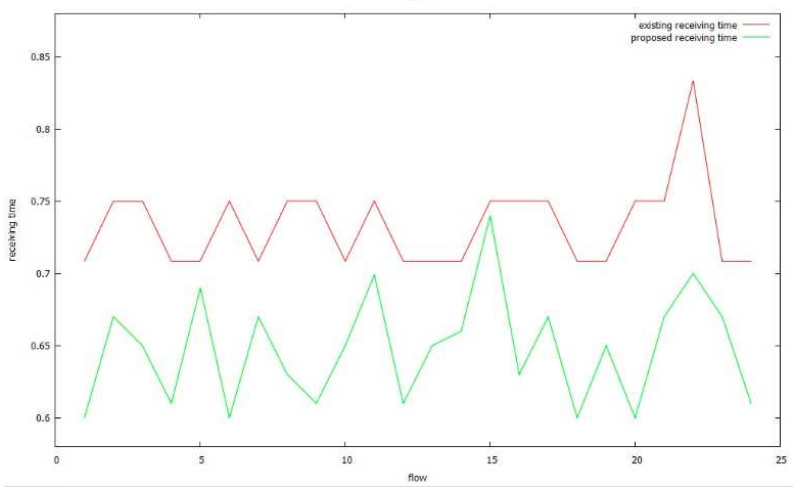

Fig. 6. Reception Time

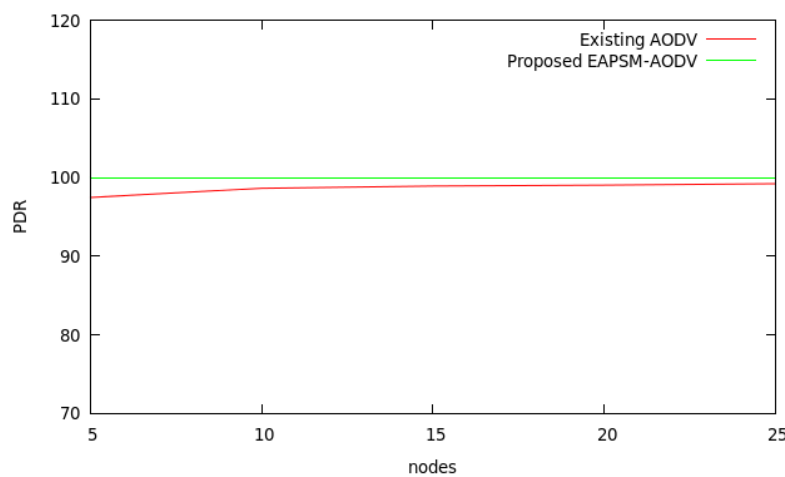

Fig. 7. Comparison of PDR under static conditions

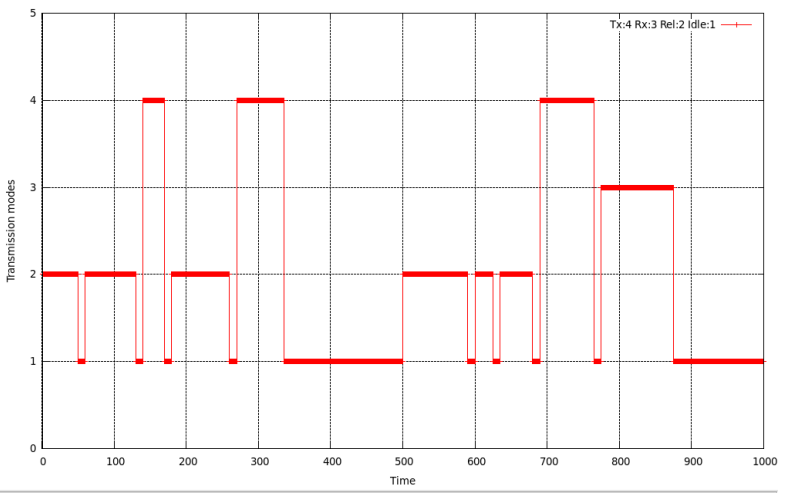

Fig. 8. Node at different mode

Figure 9 depicts the path selection of proposed and existing model. In the proposed model the nodes having maximum energy and minimum battery draining rate with Active PSM path is selected compared to existing model. This results in high throughput and PDR.

Figure 10 shows the PDR comparison between our proposed and existing models in dynamic nature of node. It can be noticed that our proposed model results in $100 \%$ PDR compared to existing model. This is due to the consideration of metrics such as remaining energy, draining rate, link duration and PSM mode of a node during routing process.

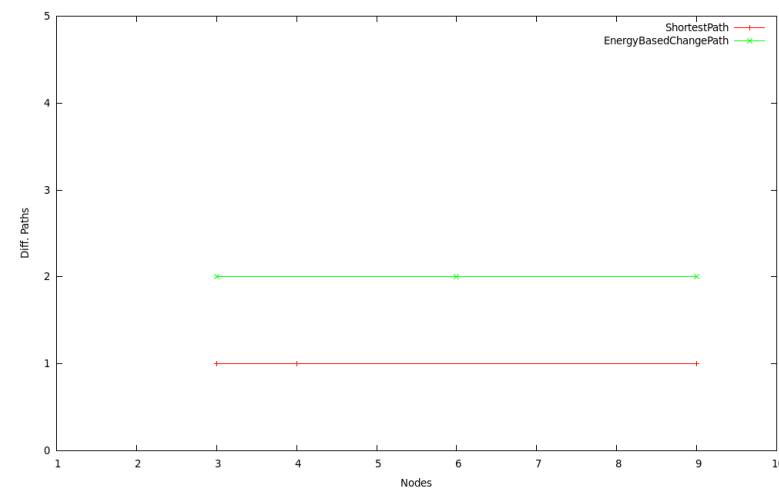

Fig. 9. Comparison of path selection in energy and shortest path based routing

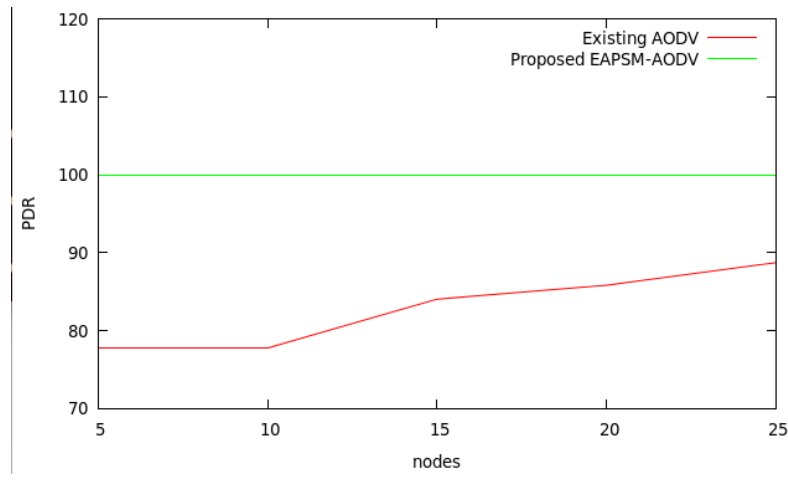

Fig. 10. Comparison of PDR under mobility conditions

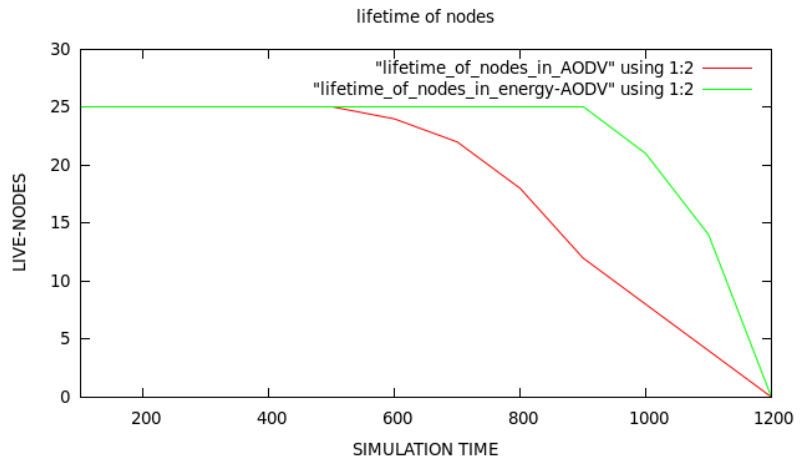

Fig. 11. Life time of network

Figure 11 depicts the lifetime of nodes variation over a certain period of time in basic AODV and EAPSM-AODV models. We can notice that in basic AODV some nodes die earlier, which leads to network failure because of repeated usage of same node whereas some other nodes lead long life because of these nodes are not used over a period of time. In EAPSM-AODV all nodes maintain same lifetime because of usage of PSM that changes its status using remaining energy and draining speed factors. From the figure we can see that all the nodes are active till $1000 \mathrm{sec}$ and all nodes get died over a period of $200 \mathrm{sec}$. So in EAPSM-AODV, all nodes balance their lifetime by considering energy factors in AODV. 


\section{CONCLUSION}

In this work we presented a novel AODV routing model that increases network lifetime by considering battery resource of node and PSM of ieee 802.11s. THe results shows that the proposed model results in $100 \%$ packet delivery ratio there by improving quality of service. The model is tested for both static and dynamic nature of nodes. RandomWayPoint mobility model is considered for moving nodes in the current work. The model will be tested using other mobility model as future work considering velocity and acceleration as parameters.

\section{ACKNOWLEDGMENT}

Part of this paper has been prepared as a contribution to the state project of the Board of the Ministry of Education of the Russian Federation (task 2.136.2014/K)

\section{REFERENCES}

[1] A.Akram and M.Shafqat. Battery and frequency optimized aodv for wireless mesh networks. Canadian Journal on Multimedia and Wireless Networks, 1:31-37, Apr 2010.

[2] Akhtar, Nakhai A.M, M.R., and Aghvami. Power aware cooperative routing in wireless mesh networks. IEEE Communications Letters, 16:226-236, may 2012.

[3] A.M. Akhtar, M.R. Nakhai, and A. Hamid Aghvami. Energyefficient adaptive routing in wireless ad hoc and mesh networks. 1:249-256, 2012.

[4] Alves, R.L.Carranoand R.Monteiro Moraes, and N.Albuquerque. Persistence of routing tables in wireless mesh networks with duty cycling. 10th IEEE Symp. on Computers and Communications (ISCC 2005), pages 21-23, 2012.

[5] IEEE2011. Ieee, draft amendment: Ess mesh networking. IEEE P802.11s Draft 12.00, Jun 2011.

[6] J.Chokhawala and A.Cheng. Real time system laboratory. optimizing power aware routing in mobile ad-hoc networks. International Journal of Computer Applications, 1, 2011.

[7] T. A. Le and M. R. Nakhai. Possible power-saving gains by dividing a cell into tiers of smaller cells. iet electronics letters. 46:1163-1165, Aug 2010.

[8] L.Romdhani and C.Bonnet. Energy consumption speed-based routing for mobile ad hoc networks. International Conference on Distributed Computing Systems Workshops 24th ICDCSW, 2004.

[9] Mhlanga, M.M.Olwal, T.OMutanga, M.B.Adigun, and M.O. Energy optimization based path selection algorithm for ieee 802.11s wireless mesh networks. IEEE AFRICON, Zambia, pages 1-6, sep 2011.

[10] P.Gupta and P.Saxena. Energy consumption in wireless ad hoc networks. Third international conference on emerging trends in engineering and technology IEEE, 2010.

[11] S.P.Shiva Prakash, T.N. Nagabhushan, and Kirill Krinkin. Energy draining rate based-aodv routing in wireless mesh network. In Proc. 12th Conference of Open Innovations Association (FRUCT), pages 179-189, 2012.

[12] S.P.Shiva Prakash, T.N. Nagabhushan, and Kirill Krinkin. Energy aware power save mode management in wireless mesh networks. In Proc. 14th Conference of Open Innovations Association (FRUCT), pages 122-131, 2013.
[13] Y.Wei, C.Wang, M.Song, Yue Ma, and X.Wang. Energy saving dynamic relaying scheme in wireless cooperative networks using markov decision process. In Proc. IEEE Vehicular Technology Conference, may 2012. 